\title{
Helicobacter pylori Infection and Gastric Mucosal Atrophy in Two Ethnic Groups in Nepal
}

\author{
Muhammad Miftahussurur ${ }^{1,2}$, Rabi Prakash Sharma ${ }^{3}$, Pradeep Krishna \\ Shrestha $^{3}$, Ramesh Kumar Maharjan ${ }^{4}$, Seiji Shiota ${ }^{1}$, Tomohisa Uchida ${ }^{5}$, Hiroki \\ Sato $^{1}$, Yoshio Yamaoka ${ }^{1,6 *}$
}

\begin{abstract}
Serum anti-Helicobacter pylori antibodies and pepsinogens (PGs) have been used as gastric cancer screening and gastric mucosal status markers. Nepal is a low risk country for gastric cancer. However, the mountainous populace in the northern region culturally linked to Tibet as well as Bhutan, a neighboring country, have a high risk of GC. We collected gastric biopsy specimens and sera from 146 dyspeptic patients living in Kathmandu, Nepal. We also examined the sera of 80 volunteers living in the mountainous regions of the Himalayas. The optimal cut-off was calculated for serum biomarkers against the histology. Kathmandu patients $(\mathbf{4 3 . 8 \% )}$ were serologically positive for $\mathrm{H}$. pylori infection, which was significantly lower than that for the mountainous $(61.3 \%$, $P=0.01)$. The same results also found in the prevalence of PG-positivity, PG I levels and PG I/II ratios $(P=0.001$, $P<0.0001$ and $P=0.03$, respectively). Moreover, the PG I/II ratios were significantly, and inversely correlated with the OLGA score $(r=\mathbf{- 0 . 3 3}, \mathrm{P}<\mathbf{0 . 0 0 9})$. The low incidence of gastric cancer in Nepal can be attributed to low gastric mucosal atrophy. However, the mountainous subjects have high-risk gastric mucosal status, which could be considered a high-risk population in Nepal.
\end{abstract}

Keywords: Helicobacter pylori - pepsinogens - gastric cancer - Nepal

Asian Pac J Cancer Prev, 16 (17), 7911-7916

\section{Introduction}

The sequence of events in the gastric mucosa that precede the manifestation of gastric cancer has been shown to begin decades earlier. Typically, it begins with chronic non-atrophic gastritis that progresses to chronic atrophic gastritis, which may eventually lead to intestinal metaplasia, dysplasia, and finally adenocarcinoma (Kuipers, 1999). Helicobacter pylori infection is accepted as the primary cause of chronic gastritis (Suerbaum and Michetti, 2002). Moreover, severe atrophic gastritis, accompanying intestinal metaplasia caused by persistent $H$. pylori infection, is closely related to the development of gastric cancer (Correa, 1992). Histological evaluation of gastric biopsy specimens is a standard method to identify atrophy. On the other hand recent reports showed that serum pepsinogens (PGs) are valuable in the serological screening of gastric diseases, including gastric atrophy and gastric cancer (Bornschein et al., 2012; Daugule et al., 2011; Leja et al., 2012; Lomba-Viana et al., 2012; Miki, 2006), particularly in Japan (Leung et al., 2008).
Pepsinogens (PGs) are aspartic proteinases that are mainly secreted by the gastric cells. They can be classified into two biochemically and immunologically distinct types: PG I and PG II (Gritti et al., 2000). Low serum PG I levels and PG I/II ratios have been associated with severe gastric atrophy, and are frequently observed in gastric cancer patients (Kim and Jung, 2010; Sipponen and Graham, 2007). In particular, it is reported that the combination of $H$. pylori serology, and the measurement of PG I levels and PG I/II ratios can be applied to gastric cancer screening in Japan (Leung et al., 2008; Miki, 2011). However, serum PG levels are affected by many factors such as the geographic area, race, age, gender, smoking and drinking habits, and H. pylori infection (Ang et al., 2005; Bornschein et al., 2012; Kikuchi et al., 1995). Therefore, the cut-off value for PG levels can vary with geographic locations. Meta-analyses showed that a PG I level $\leq 70 \mathrm{ng} / \mathrm{mL}$, and PG I/II ratio $\leq 3$ had a sensitivity of $57 \%$, specificity of $80 \%$, positive predictive value (PPV) of $15 \%$, and negative predictive value (NPV) of $83 \%$ in the screening for atrophic gastritis (Miki, 2006).

${ }^{1}$ Department of Environmental and Preventive Medicine, ${ }^{5}$ Department of Molecular Pathology, Oita University Faculty of Medicine, Yufu, Japan, ${ }^{2}$ Gastroentero-Hepatology Division, Department of Internal Medicine, Airlangga University Faculty of Medicine, Surabaya, Indonesia, ${ }^{3}$ Gastroenterology Department, Maharajgunj Medical Campus, Tribhuvan University Teaching Hospital, ${ }^{4}$ Shechen Clinic \& Hospice, Boudha, Kathmandu, Nepal, ${ }^{6}$ Department of Gastroenterology and Hepatology, Baylor College of Medicine and Michael DeBakey Veterans Affairs Medical Center, Houston, Texas, USA*For correspondence: yyamaoka@oita-u.ac.jp 
Nepal is a small landlocked country in South Asia, located in the Himalayas. Kathmandu, its capital, has witnessed several different waves of human migrations. Therefore, it is not surprising that the indigenous inhabitants, the Newars, are postulated to be a mixture of Austro-Asiatic, Dravidian, Indo-Mongoloid, and Aryan origins (Gayden et al., 2013). Although genetic analyses revealed that the Kathmandu ancestry is a combination of East and South Central Asian lineages (Gayden et al., 2013), the age-standardized incidence rate (ASR) of gastric cancer in Nepal (5.3 cases/100,000 population/year) is similar to that of South Asian countries (India and Bangladesh). However, it is much lower than that of East Asian countries such as Japan and China (6.1 ,5.8, 29.9, and 22.7/100,000, respectively) (International Agency for Research on Cancer, GLOBOCAN 2012; http://globocan.iarc.fr).

The mountainous people of northern Kathmandu live in temples temporarily during winter, and interestingly, are culturally linked to the Buddhists of Tibet. Our previous report showed that Bhutan, a neighboring country with high prevalence of $H$. pylori infection and gastric mucosal atrophy, is also culturally linked to the Tibetan Buddhists (Shiota et al., 2013). Since the Kathmandu population incorporate both the Buddhist and Hindu traditions (Gayden et al., 2013), it would be interesting to investigate the association between $H$.pylori infection and gastric atrophy in these populations. Toward this, we also calculated the best cut-off and predictive values for discriminating atrophic gastritis based on PGs in the Kathmandu subjects. We hypothesized that because of the Tibetan connection, the prevalence of $H$. pylori infection and/or advanced gastric atrophy is higher in the mountainous population, compared to that of Kathmandu.

\section{Materials and Methods}

\section{Study populations and sample collection}

In December 2013, we recruited 146 dyspeptic patients living in Kathmandu from the endoscopy services of the Gastroenterology department, Tribhuvan University Teaching Hospital (TUTH), Kathmandu. Written informed consent was obtained from all the participants, and the study protocol was approved by the Ethics Committees of TUTH and Oita University Faculty of Medicine, Japan. To measure the sensitivity and specificity of the enzymelinked immunosorbent assay (ELISA) kit for PGs, and to calculate the best cut-off values for discriminating atrophic gastritis based on PGs, we collected blood samples on the day of endoscopy. Experienced endoscopists acquired two gastric biopsy specimens during each endoscopy procedure: one from the lesser curvature of the antrum approximately $3 \mathrm{~cm}$ from the pyloric ring, and another from the greater curvature of the corpus used for histological examination. We also examined the sera of 80 volunteers, living in the mountainous regions of the Himalayas, but staying during winder at the Boudhanath (Boudha) temple, Kathmandu. In addition, we also obtained information on the smoking and drinking habits in both these populations.
Serological analyses of $H$. pylori infection and PG status

The blood samples were centrifuged within $1 \mathrm{~h}$ after collection. The separated sera were used for measurement of the $H$. pylori antibody titers and PG levels. The anti- $H$. pylori IgG levels were quantified using an ELISA kit (Eiken, Co. Ltd., Tokyo, Japan), and the PG I and II levels were measured using Pepsinogen ELISA (Eiken, Co. Ltd), according to the manufacturers' instructions. Individuals with serum $H$. pylori antibody titers $\geq 10 \mathrm{U} / \mathrm{mL}$ were classified as $H$. pylori-positive (per the manufacturer's instructions); those with PG I levels $\leq 70 \mathrm{ng} / \mathrm{mL}$, and PG $\mathrm{I} / \mathrm{II}$ ratios $\leq 3.0$ were classified as PG-positive (according to the Japanese guidelines) (Miki, 2011). Next, the subjects were categorized into four groups according to the $H$. pylori and PG sero-positivity (called the ABC method) (Miki, 2011): group A (H. pylori-negative/PG-negative), group B (H. pylori-positive/PG-negative), group C $(H$. pylori-positive/PG-positive), and group D (H. pylorinegative/PG-positive).

\section{Histology}

All the biopsy tissues were fixed in $10 \%$ buffered formalin for $24 \mathrm{~h}$, and embedded in paraffin. Serial sections were then stained with hematoxylin and eosin, and May-Giemsa stain. The status of the gastric mucosa was evaluated according to the updated Sydney system (Dixon et al., 1996). The degree of atrophy was classified into four grades: 0 , normal; 1 , mild; 2 , moderate; and 3, marked. Samples of grade 1 or more were considered atrophy-positive, according to a previous report (Bornschein et al., 2012). In addition, the stage of gastritis was assessed according to the Operative Link for Gastritis Assessment (OLGA) system (Rugge et al., 2005; Rugge et al., 2007). To minimize the potential for bias, an experienced pathologist (TU), who analyzed histological data for the Myanmar, Vietnam, Bhutan, and Dominican Republic studies (Nguyen et al., 2010a; Nguyen et al., 2010b; Shiota et al., 2014; Shiota et al., 2010; Vilaichone et al., 2013), evaluated all the specimens in this study.

\section{Data analyses}

Discrete variables were tested using the chi-square test; continuous variables were tested using the MannWhitney U and t-tests. The Spearman rank coefficients (r) were determined to evaluate the association between the PGs and gastric mucosal atrophy. A multivariate logistic regression model was used to calculate the odds ratios (OR) of the clinical outcomes by including the age, sex, $H$. pylori infection, and gastritis type. All determinants with $P$ values $<0.10$ were entered together in the full model of logistic regression, and the model was reduced by excluding variables with $P$ values $>0.10$. The $\mathrm{OR}$ and $95 \%$ confidence interval (CI) were used to estimate the risk. A P value $<0.05$ was accepted as statistically significant. Receiver-operating curves (ROC) were used to calculate the best cut-off and predictive values for discriminating atrophic gastritis based on the PG I/II ratios. The SPSS statistical software package version 18.0 (SPSS, Inc., Chicago, IL, USA) was used for all statistical analyses. 


\section{Results}

$H$. pylori infection rate in the dyspeptic patients

We recruited 146 dyspeptic patients living in Kathmandu (76 females and 70 males; mean age $42.2 \pm 15.7$ years). Our serological examinations revealed that among the 146 patients, 64 (43.8\%) were positive for H. pylori infection. The H. pylori infection rate by age group was $37.5 \%$ (12/32), 38.2\% (13/34), 55.9\% (19/34), $44.0 \%$ $(11 / 25)$, and $42.9 \%(9 / 21)$ for patients aged $\leq 29,30-39$, $40-49,50-59$, and $\geq 60$ years, respectively (Figure 1 ).

We also examined the sera of 80 volunteers living in the mountainous regions of the Himalayas (38 females and 42 males; mean age $43.5 \pm 16.7$ years). Interestingly, our serological data showed that 49 of the $80(61.3 \%)$ volunteers were positive for $H$. pylori infection, which was significantly higher than that for the Kathmandu patients $(\mathrm{P}=0.01)$. The mean age was significantly lower for the mountainous subjects who were H.pylori-positive, than for those who were H.pylori-negative $(\mathrm{P}<0.0001)$. Furthermore, a high prevalence of $H$. pylori infection was detected in the younger age groups ( $\leq 29$ years) for the mountainous volunteers $(85 \%, 17 / 20)$, compared with the Kathmandu subjects $(\mathrm{P}=0.003)$. However, in both the populations, there was no statistically significant relation between the $H$. pylori infection rate and age $(\mathrm{P}=0.42$ and $\mathrm{P}=0.15$, respectively). Additionally, while smoking habit was significantly higher in the Kathmandu, rather than the

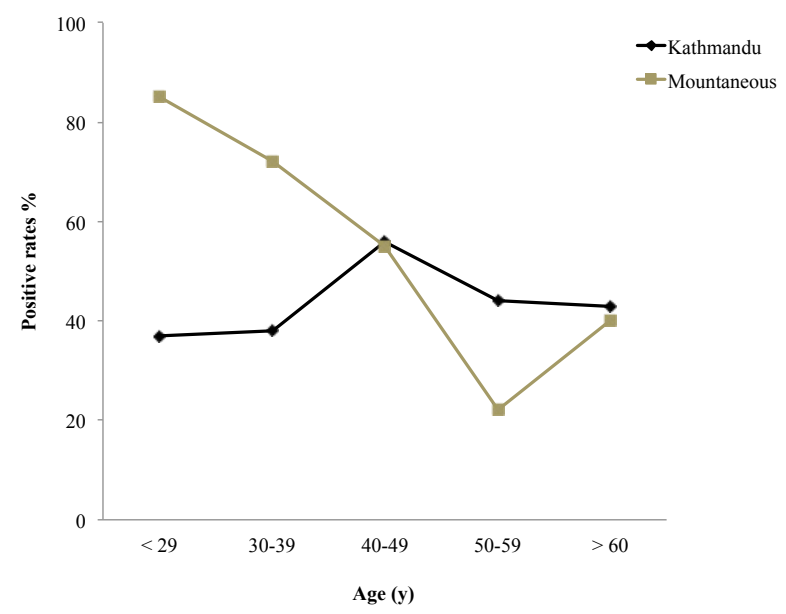

Figure 1. Age-related Prevalence of $\boldsymbol{H}$.pylori infection in 146 Kathmandu and 80 mountainous subjects in Nepal. $H$. pylori sero-positivity was determined by ELISA. Individuals with serum $H$.pylori antibody titers $>10 \mathrm{U} / \mathrm{mL}$ were classified as $H$. pylori-positive. Sero-positivity for $H$. pylori infection in the Kathmandu subjects was lower than that in the mountainous subjects mountainous subjects $[26.7 \%$ (39/146) versus $7.5 \%$ (6/80), $\mathrm{P}=0.001]$, no significant difference in the drinking habits of the two populations was found $(\mathrm{P}>0.05)$. Unfortunately, we were permitted to perform endoscopy only in the Kathmandu subjects; therefore, we could measure the sensitivity and specificity of the ELISA kit compared with histology as a gold standard for Kathmandu population. The sensitivity and specificity were $89.1 \%$ and $83.5 \%$, respectively.

\section{Status of PGs and mucosal atrophy in Nepal}

According to Japanese standards, we defined the PGpositive status by cut-off values of $\leq 70 \mathrm{ng} / \mathrm{mL}$ and $\leq 3.0$ for the PG I levels and PG I/II ratios, respectively. Based on this, the overall prevalence of PG-positive subjects in the Kathmandu population was $11.6 \%$ (17/146), significantly higher in the $H$. pylori-positive than in the $H$. pylorinegative patients $[18.8 \%(12 / 64)$ versus $6.1 \%(5 / 82)$, $\mathrm{P}=0.02]$. The $\mathrm{PG}$ II levels were also significantly higher, while the PG I/II ratios were significantly lower in the $H$. pylori-positive, than in the $H$. pylori-negative patients ( $\mathrm{P}$ $<0.0001$ for both the cases) (Table 1). In contrast, there was no difference in the PG I levels between the H.pyloripositive and -negative patients $(\mathrm{P}=0.44)$.

Among the 17 PG-positive patients from Kathmandu, $15(88.2 \%)$ had atrophy in the antrum (Table 2). Although the proportion was significantly higher in the PG-positive, than in the -negative group $(\mathrm{P}=0.02), 77$ of the $129 \mathrm{PG}-$ negative patients $(59.7 \%)$ also had atrophy in the antrum. PG-positive/atrophy-negative subjects were also found atrophy in $77.8 \%(14 / 18)$ in the corpus. Therefore, using the Japanese criteria in our Nepalese study yielded highly false-negative results.

Next, we examined the correlation between the severity of gastric mucosal atrophy and PG levels (Table 3). Although the PG I levels did not correlate with the atrophy scores $(\mathrm{P}=0.21$ in the antrum and $\mathrm{P}=0.07$ in the

Table 2. Association between PG Status and Mucosal Atrophy in the Antrum and Corpus in the Kathmandu Population

\begin{tabular}{lccc}
\hline & $\begin{array}{c}\text { Atrophy-positive } \\
\mathrm{n}(\%)\end{array}$ & $\begin{array}{c}\text { Atrophy-negative } \\
\mathrm{n}(\%)\end{array}$ & $\begin{array}{c}\text { Total } \\
(\mathrm{n})\end{array}$ \\
\hline Antrum & & & \\
PG-positive & $15(88.2)$ & $2(11.8)$ & 17 \\
$\quad$ PG-negative & $77(59.7)$ & $52(40.3)$ & 129 \\
Total & $92(63.0)$ & $54(37.0)$ & 146 \\
Corpus & & & \\
$\quad$ PG-positive & $4(22.2)$ & $14(77.8)$ & 18 \\
PG-negative & $11(8.6)$ & $117(91.4)$ & 128 \\
Total & $14(9.7)$ & $131(90.3)$ & 146 \\
\hline
\end{tabular}

Table 1. Characteristics of PGs in the Kathmandu and Mountainous Populations

\begin{tabular}{|c|c|c|c|c|c|c|}
\hline & Sero-positive & $\begin{array}{l}\text { Kathmandu } \\
\text { Sero-negative }\end{array}$ & $P$ value & Sero-positive & $\begin{array}{l}\text { Mountainous } \\
\text { Sero-negative }\end{array}$ & $P$ value \\
\hline $\mathrm{n}$ & 64 & 82 & - & 49 & 31 & - \\
\hline PG I (ng/mL) & $104.2+101.6 *$ & $95.4+70.4$ & 0.64 & $55.0+22.5$ & $55.6+33.0$ & 0.44 \\
\hline PG II (ng/mL) & $31.1+23.8 *$ & $19.1+13.2$ & $<0.0001$ & $19.9+8.8$ & $13.9+9.0$ & $<0.0001$ \\
\hline PG I/II ratio & $3.5+1.3 *$ & $5.0+1.5$ & $<0.0001$ & $2.9+1.1$ & $4.3+1.4$ & $<0.0001$ \\
\hline PG-positive (n) & $12(18.8 \%)^{*}$ & $5(6.1 \%)$ & 0.02 & $24(49.0 \%)$ & $0.0(0 \%)$ & $<0.0001$ \\
\hline
\end{tabular}

$* \mathrm{P}<0.05$ compared to the mountainous subjects 
corpus), the PG I/II ratios showed a significant inverse correlation in the antrum and corpus $(\mathrm{P}<0.0001, \mathrm{r}=-0.46$; and $\mathrm{P}=0.002, \mathrm{r}=-0.25$, respectively). Moreover, when we analyzed only the $H$. pylori-positive patients, the PG I/ II ratios showed a significant inverse correlation with the atrophy score in the antrum $(\mathrm{P}=0.002, \mathrm{r}=-0.38)$, but not in the corpus $(\mathrm{P}=0.27)$. We also examined the correlation between the OLGA score and the severity of atrophy in the H. pylori-positive Kathmandu subjects. Only the PG I/II ratio was significantly, and inversely correlated with the OLGA score $(r=-0.33, \mathrm{P}<0.009)$.

To evaluate the predictive factors for the presence of atrophy, we performed a multivariate analysis. In the antrum, only the $H$. pylori infection was an independent risk factor for the development of atrophy $(\mathrm{OR}=9.75$, 95\% CI, 3.9 to 24.2). Age and H. pylori infection were also significantly associated with atrophy in the corpus $[\mathrm{OR}=1.0695 \% \mathrm{CI}, 1.0$ to $1.1 ; \mathrm{OR}=5.5,95 \% \mathrm{CI}, 1.4$ to 22.2, respectively).

Comparison of PG levels between the two ethnic groups are shown in Table 1. Among the H.pylori-positive subjects, the PG I and II levels were significantly higher in the Kathmandu, than in the mountainous subjects ( $P$ $<0.0001$ and $\mathrm{P}=0.003$, respectively). Furthermore, the $\mathrm{PG}$ $\mathrm{I} / \mathrm{II}$ ratios were significantly lower in the mountainous than in the Kathmandu subjects $(\mathrm{P}=0.03)$. The overall prevalence of PG-positivity in the Kathmandu population was lower than that in the mountainous subjects $(\mathrm{P}=0.001)$, and the differences were especially significant in the age groups of $<29$ and $40-49$ years ( $\mathrm{P}=0.001$ for both).

The distribution of four groups in each age category, according to the $\mathrm{ABC}$ method, is shown in Figure 2. In the Kathmandu subjects, Group A $(77 / 146,52.7 \%)$ was the most dominant, followed by groups B (52/146, 35.6\%), C $(12 / 146,8.2 \%)$, and D $(5 / 146,3.4 \%)$. Despite the groups A $(31 / 80,38.8 \%)$ and B $(25 / 80,31.3 \%)$ being the most dominant, high proportions of the mountainous subjects belonged to group C $(24 / 80,30 \%)$. This was consistent with the results obtained for PGs. In fact, this proportion was significantly higher than that in the Kathmandu subjects $(\mathrm{P}=0.007)$. The proportional distribution of the PG-positive subjects in the $H$. pylori-positive group was $37.5 \%, 16.7 \%, 20.8 \%, 0.0 \%$, and $25.0 \%$ for the age categories of $<29,30-39,40-49,50-59$, and $>60$ years, respectively.

\section{The best cut-off value for PGs}

There was no correlation between the PG I levels and the OLGA scores. When we used the cut-off value for the PG I/II ratios as $\leq 3.0$, the sensitivity, specificity, PPV, and NPV were $30.4 \%, 94.4 \%, 90.3 \%$, and $44.4 \%$, respectively, for stages higher than I (using the OLGA score as the gold standard for histology). In cases higher than stage II in the OLGA score, they were $46.7 \%, 81.7 \%, 22.6 \%$, and $93.8 \%$, respectively.

Since the low sensitivity was due to a large number of false-negatives, we determined the best cut-off value of PG I/II with an ROC curve. The ROC curve for discriminating atrophic gastritis, based on PG I/II ratios, in the Kathmandu patients is shown in Figure 3. For stages more than or equal I in the OLGA score, regarded
Table 3. The levels of PG I, PG II, and PG I/II in Atrophic Gastritis (mean \pm SD) in the Kathmandu Population

\begin{tabular}{llrlll}
\hline & Grade & $\mathrm{n}$ & PG I & PG II & PG I/II \\
\hline Antrum & 0 & 54 & $94.7 \pm 72.6$ & $18.3 \pm 12.9$ & $5.2 \pm 1.5$ \\
& 1 & 77 & $105.7 \pm 98.2$ & $28.1 \pm 23.0$ & $4.0 \pm 1.5$ \\
& 2 & 14 & $85.6 \pm 50.4$ & $27.2 \pm 13.3$ & $3.1 \pm 1.1$ \\
& 3 & 1 & 42.7 & 13.8 & 3.1 \\
Corpus & 0 & 131 & $99.3 \pm 88.7$ & $23.5 \pm 19.7$ & $4.5 \pm 1.6$ \\
& 1 & 11 & $114.8 \pm 46.6$ & $36.7 \pm 15.1$ & $3.3 \pm 1.1$ \\
& 2 & 4 & $52.2 \pm 20.4$ & $19.8 \pm 3.7$ & $2.6 \pm 0.6$ \\
& 3 & 0 & $\mathrm{NA}$ & $\mathrm{NA}$ & $\mathrm{NA}$ \\
OLGA & 0 & 54 & $94.7 \pm 72.6$ & $18.3 \pm 12.9$ & $5.2 \pm 1.5$ \\
& $\mathrm{I}$ & 77 & $105.7 \pm 98.2$ & $28.2 \pm 23.0$ & $4.0 \pm 1.5$ \\
& II & 13 & $89.6 \pm 50.1$ & $28.0 \pm 13.6$ & $3.2 \pm 1.0$ \\
& III & 2 & $38.1 \pm 6.5$ & $15.9 \pm 2.9$ & $2.5 \pm 0.8$ \\
& IV & 0 & $\mathrm{NA}$ & $\mathrm{NA}$ & $\mathrm{NA}$ \\
\hline
\end{tabular}

OLGA, Operative Link on Gastritis Assessment; NA, not applicable

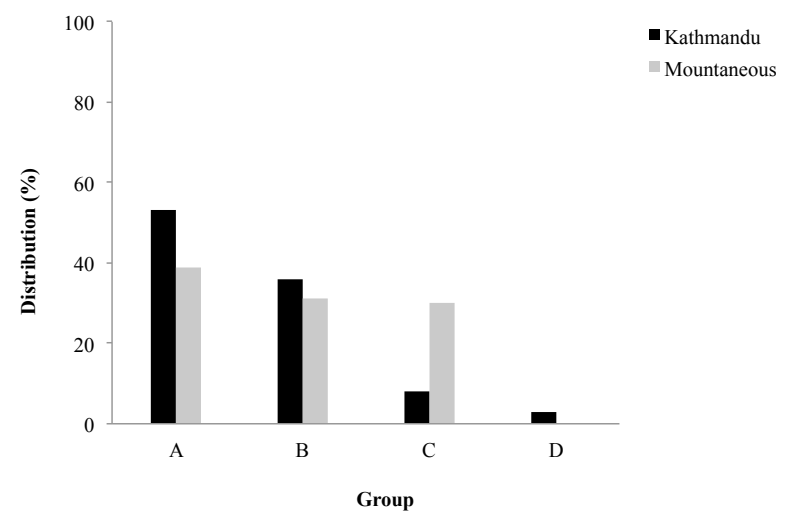

Figure 2. Distribution of $\boldsymbol{H}$. pylori Infection and PG Status among the Kathmandu and Mountainous Subjects. The subjects were classified into four groups according to the results of the two serological tests for $H$.pylori and PG detection: group A (H. pylori-negative/PG-negative), group B (H. pylori-positive/PG-negative), group C (H. pyloripositive/PG-positive), and group D (H. pylori-negative/ PG-positive). Group $\mathrm{C}$ in the mountainous subjects claim a high proportion, which is significantly higher than that in the Kathmandu subjects

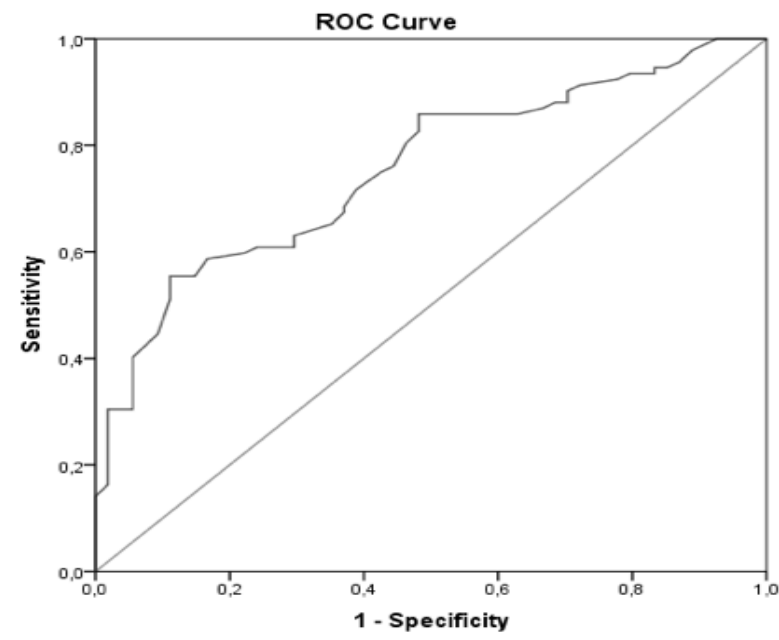

Figure 3. ROC Curve for PG I/II in the Kathmandu Population for Stages Higher than I. The optimal cut-off points for the PG I/II ratios were determined by analyzing the receiver-operating characteristic (ROC) 
Helicobacter pylori and Gastric Mucosal Atrophy in Two Ethnic Populations in Nepal

as atrophy-positive, the best cut-off value of PG I/II was 4.65 (the sensitivity, specificity, PPV, and NPV were $71.7 \%, 61.1 \%, 75.9 \%$, and $55.9 \%$, respectively). The area under curve (AUC) was 0.754 (95\% CI, 0.676-0.832). For stages more than or equal II in the OLGA score, regarded as atrophy-positive, the best cut-off value of PG I/II was 3.45 (the sensitivity, specificity, PPV, and NPV were $86.7 \%, 66.4 \%, 22.8 \%$, and $97.8 \%$, respectively), and the AUC was 0.758 (95\% CI, 0.641-0.874).

\section{Discussion}

Based on the ASR of gastric cancer, Asian countries can be categorized as high-risk (e.g., Japan, South Korea, and China), intermediate-risk (e.g., Vietnam), or low-risk (e.g., India and Nepal) (Ferlay et al., 2010). Previous studies confirmed that Nepal is part of the Asian enigma: despite the high $H$. pylori infection rate, the incidence of gastric cancer is low (Malaty, 2007; Miwa et al., 2002). In this study, we found by serology that the prevalence of $H$. pylori infection is $43.8 \%$ in Kathmandu, with low gastric mucosal atrophy. This result is similar to our unpublished data $(38.4 \%)$, where we measured the prevalence of $H$. pylori infection in a Kathmandu population by the combination of three diagnostic tests [culture, rapid urease test (RUT), and histology confirmed by immunohistochemistry (IHC)], and at least one of the tests yielded positive results. We also found that the source of drinking water and religion were associated with the prevalence of $H$. pylori infection. In this study, we found that the ethnic Nepalese people who are culturally linked to the Buddhists of Tibet (mountainous population) have higher prevalence of $H$. pylori infection $(61.3 \%)$, compared with the majority of the general Nepalese population. Interestingly, in a previous study, authors examined the $H$. pylori infection rate in a minor indigenous ethnic population of the Eastern Himalayas (Sherpa), using a stool antigen test, and found results similar to ours (70.5\%) (Sherpa et al., 2012). Further, in our previous study, we found that the overall prevalence of $H$. pylori infection in Bhutan, a neighboring country, was $73.4 \%$, where subjects were considered $H$. pyloripositive if at least one of the tests (RUT, culture, histology confirmed by IHC, or serology) showed positive results (Vilaichone et al., 2013). The differential risk of gastric cancer between Nepal and Bhutan may be attributed to major ethnic genetic variations.

In this study, we examined the application of an ELISA kit (manufactured by Eiken Company, Japan; based on a Japanese $H$. pylori strain) for the detection of $H$. pylori infection in Nepal. Although H. pylori antibody titers vary greatly depending on the test kit used (Burucoa et al., 2013), we found the sensitivity and specificity of the ELISA kit to be $89.1 \%$ and $83.5 \%$, respectively, for the Kathmandu population by histology as the gold standard. The results were comparable with those obtained in Japan, where the sensitivity and specificity of the kit were reported to be $95.2-100 \%$ and $76.2-80.0 \%$, respectively (Matsuo et al., 2001). However, for future studies, it would be preferable to develop an ELISA kit using $H$. pylori strains indigenous to Nepal.
Serum PG I and II are upregulated upon H. pylori infection. However, because PG II levels exhibit a greater increase than those of PG I, the PG I/II ratio decreases in the presence of H.pylori. Low PG I levels and PG I/II ratios have been associated with severe gastric atrophy, and are frequently observed in gastric cancer patients (Kim and Jung, 2010; Miki, 2011). In Kathmandu, the PG I/II ratio showed significant inverse correlation with the atrophy score in the antrum, as well as the OLGA score. Interestingly, by using PG I levels PG I/II ratios and PG-positivity as markers for atrophy, we found that the mountainous population has higher atrophy than that of Kathmandu. The ABC method also showed that a high proportion of the mountainous subjects belonged to group C $(30 \%)$ compared with that of Kathmandu, the PG-positivity rates being even higher in the younger subjects of this population. The results were similar to those of our previous study in Bhutan (Shiota et al., 2013). In the Bhutanese population, the rates of group B $(53.8 \%)$ and C $(17.3 \%)$ were higher than those in Japan [group B (16.4\%) and group C (9.3\%)] (Miki, 2011). Moreover, the prevalence of PG-positivity in the mountainous population $(30 \%)$ was much higher than that in the Bhutanese subjects $(19.4 \%)$. These results suggest that the mountainous people have high-risk gastric mucosal status, and might be a high-risk population in Nepal. Further studies of host and bacterial factors in the mountainous population are necessary to better elucidate reasons behind the differential prevalence of $H$. pylori infection and/or gastric atrophy risk in these populations.

Unfortunately, we were not permitted to obtain gastric biopsy specimens from the mountainous subjects. In Kathmandu, we could use histology as gold standard. The best cut-off values for the PG I/II ratios were 4.65 and 3.45, for stages higher than I and II, respectively, in the Kathmandu population only. In addition, we included patients from Kathmandu with dyspeptic symptoms, but only volunteers from the mountainous population. Broadly, the prevalence of $H$. pylori infection is higher in dyspeptic patients than in the general population. However, the prevalence of $H$. pylori infection is higher in the mountainous, than in the Kathmandu population, even when only volunteers were included from the mountainous population. Further multi-center studies, recruiting subjects on a large scale from various ethnicities, are required to confirm our findings.

In conclusion, the low incidence of gastric cancer in Nepal can be attributed to low gastric mucosal atrophy. Serum PG levels were associated with mucosal atrophy in Nepal, similar to that in other countries. However, the mountainous population has high-risk gastric mucosal status, which might be considered a high-risk population. Our analyses also showed that $H$. pylori infection is an independent risk factor for the development of atrophy, both in the antrum and corpus. These results lay stress on the need for H. pylori eradication in Nepal.

\section{Acknowledgements}

This work was supported in part by grants from the National Institutes of Health (DK62813) and the Grants-in- 
Aid for Scientific Research from the Ministry of Education, Culture, Sports, Science, and Technology(MEXT) of Japan (24406015, 24659200, 25293104, 26640114 and 15H02657) (YY). It was also supported by the Japan Society for the Promotion of Science (JSPS) Institutional Program for Young Researcher Overseas Visits (YY), the Strategic Funds for the Promotion of Science and Technology from Japan Science and Technology Agency (JST) (YY), as well as the MEXT KAKENHI (221S0002) (YY). MM is a PhD student supported by The Japanese Government (Monbukagakusho: MEXT) Scholarship Program for 2012.

\section{References}

Ang TL, Fock KM, Dhamodaran S, et al (2005). Racial differences in Helicobacter pylori, serum pepsinogen and gastric cancer incidence in an urban Asian population. $J$ Gastroenterol Hepatol, 20, 1603-9.

Bornschein J, Selgrad M, Wex T, et al (2012). Serological assessment of gastric mucosal atrophy in gastric cancer. BMC Gastroenterol, 12, 10.

Burucoa C, Delchier JC, Courillon-Mallet A, et al (2013). Comparative evaluation of 29 commercial Helicobacter pylori serological kits. Helicobacter, 18, 169-9.

Correa P (1992). Human gastric carcinogenesis: a multistep and multifactorial process--first american cancer society award lecture on cancer epidemiology and prevention. Cancer research, 52, 6735-40.

Daugule I, Sudraba A, Chiu HM, et al (2011). Gastric plasma biomarkers and Operative Link for Gastritis Assessment gastritis stage. European journal of gastroenterology \& hepatology, 23, 302-7.

Dixon M, Genta R, Yardley J, et al (1996). Classification and grading of gastritis. The updated Sydney System. International Workshop on the Histopathology of Gastritis, Houston 1994. Am J Surg Pathol, 20, 1161-81.

Ferlay J, Shin HR, Bray F, et al (2010). Estimates of worldwide burden of cancer in 2008: GLOBOCAN 2008. Int J Cancer, 127, 2893-917.

Gayden T, Perez A, Persad PJ, et al (2013). The Himalayas: barrier and conduit for gene flow. Am J Phys Anthropol, 151, 169-82.

Gritti I, Banfi G, Roi GS, et al (2000). Pepsinogens: physiology, pharmacology pathophysiology and exercise. Pharmacological research : the official journal of the Italian Pharmacological Society, 41, 265-81.

Kikuchi S, Inaba Y, Wada O, et al (1995). The association of smoking and drinking habits with serum pepsinogens. International J Epidemiol, 24, 346-53.

Kim N, Jung HC (2010). The role of serum pepsinogen in the detection of gastric cancer. Gut Liver, 4, 307-19.

Kuipers EJ (1999). Review article: exploring the link between Helicobacter pylori and gastric cancer. Alimentary pharmacology \& therapeutics, 13, 3-11.

Leja M, Cine E, Rudzite D, et al (2012). Prevalence of Helicobacter pylori infection and atrophic gastritis in Latvia. European journal of gastroenterology \& hepatology, 24, 1410-7.

Leung WK, Wu MS, Kakugawa Y, et al (2008). Screening for gastric cancer in Asia: current evidence and practice. Lancet Oncol, 9, 279-287.

Lomba-Viana R, Dinis-Ribeiro M, Fonseca F, et al (2012). Serum pepsinogen test for early detection of gastric cancer in a European country. European journal of gastroenterology \& hepatology, 24, 37-41.
Malaty HM (2007). Epidemiology of Helicobacter pylori infection. Best practice \& research. Clinical gastroenterology, 21, 205-14.

Matsuo K, Hamajima N, Suzuki T, et al (2001). Better ROC curves for a regionally developed Helicobacter pylori antibody test. Asian Pac J Cancer Prev, 2, 155-156.

Miki K (2006). Gastric cancer screening using the serum pepsinogen test method. Gastric Cancer, 9, 245-53.

Miki K (2011). Gastric cancer screening by combined assay for serum anti-Helicobacter pylori IgG antibody and serum pepsinogen levels - "ABC method". Proc Jpn Acad Ser B Phys Biol Sci, 87, 405-14.

Miwa H, Go MF, Sato N (2002). H. pylori and gastric cancer: the Asian enigma. The American journal of gastroenterology, 97, 1106-12.

Nguyen LT, Uchida T, Tsukamoto Y, et al (2010a). Clinical relevance of cagPAI intactness in Helicobacter pylori isolates from Vietnam. European journal of clinical microbiology \& infectious diseases : official publication of the European Society of Clinical Microbiology, 29, 651-60.

Nguyen TL, Uchida T, Tsukamoto Y, et al (2010b). Helicobacter pylori infection and gastroduodenal diseases in Vietnam: a cross-sectional, hospital-based study. BMC gastroenterology, 10, 114 .

Rugge M, Genta RM, Group O (2005). Staging gastritis: an international proposal. Gastroenterology, 129, 1807-8.

Rugge M, Meggio A, Pennelli G, et al (2007). Gastritis staging in clinical practice: the OLGA staging system. Gut, 56, 631-6.

Sherpa TW, Sherpa KT, Nixon G, et al (2012). The prevalence of Helicobacter pylori infection in Sherpa residents of the Upper Khumbu, an isolated community in Eastern Nepal. $N Z$ Med J, 125, 30-7.

Shiota S, Cruz M, Abreu JA, et al (2014). Virulence genes of Helicobacter pylori in the Dominican Republic. Journal of medical microbiology, 63, 1189-96.

Shiota S, Mahachai V, Vilaichone RK, et al (2013). Seroprevalence of Helicobacter pylori infection and gastric mucosal atrophy in Bhutan, a country with a high prevalence of gastric cancer. Journal of medical microbiology, 62, 1571-78.

Shiota S, Murakami K, Fujioka T, et al (2010). Populationbased strategies for Helicobacter pylori-associated disease management: a Japanese perspective. Expert review of gastroenterology \& hepatology, 4, 149-6.

Sipponen P, Graham DY (2007). Importance of atrophic gastritis in diagnostics and prevention of gastric cancer: application of plasma biomarkers. Scand J Gastroenterol, 42, 2-10.

Suerbaum S, Michetti P (2002). Helicobacter pylori infection. The New England journal of medicine, 347, 1175-86.

Vilaichone RK, Mahachai V, Shiota S, et al (2013). Extremely high prevalence of Helicobacter pylori infection in Bhutan. World journal of gastroenterology : WJG, 19, 2806-10. 